\title{
SOBRE A CLASSIFICAÇÃO DE SISTEMAS DE EQUAÇÕES DIFERENCIAIS PARCIAIS
}

Hamilton Faria Leckar Rubens Sampaio ${ }^{2}$

\section{INTRODUÇÃO}

Por que classificar equações diferenciais parciais ou problemas que as envolvem?

Dentre inúmeras questões que têm ocupado a atenção de matemáticos, engenheiros, físicos e sobretudo os especialistas na área de EDP, tanto no aspecto teórico quanto prático, talvez a mais comum seja a questão da existência, unicidade e dependência contínua de soluções com respeito aos dados, isto é, se o problema dado é bem posto no sentido de Hadamard. Mais recentemente tem merecido grande atenção a questão de saber em que sentido, um problema não bem posto no sentido acima, pode descrever situações de interesse, tanto de natureza teórica quanto prática.

Há alguns anos atrás, em relação com alguns problemas de Elasticidade Linear com Vínculo, deparamo-nos com algumas equações, de formulações simples e bem fundamentadas teoricamente, mas que não se encaixavam nas classificações que conhecíamos. Decidimos investigar, mais a fundo o problema, mas este nos pareceu requerer mais esforço que o que estavamos dispostos a empenhar. Os resultados são ainda inconclusos e este trabalho, que consideramos incompleto, visa interessar outros pesquisadores a se dedicarem ao tema.

Mais explicitamente, é sabido por todos que uma equação diferencial parcial semilinear de segunda ordem cuja incógnita é uma função real de duas ou mais variáveis (coeficientes dos termos de segunda ordem dependem apenas das variáveis independentes), pode localmente ser transformada por meio de uma mudança de variávềis independentes numa forma canônica que se relaciona, no caso de duas

Departamento de Matemática Aplicada-UFF, Rua Mário Santos Braga, s/n, 24020-140 Niterói (RJ), Email: gmahafl@vm.uff.br, hfleckar@vm.uff.br; Fax: 55217178269.

2 Departamento de Engenharia Mecânica-PUC-Rio, Rua Marquês de São Vicente, 225, 22453-900 Río de Janeiro (RJ), E-mail: rsampaio@mec.puc-rio.br; Homepage: http://www.mec.puc-río.br/prof/rsampaio/ rsampaio.html; Fax: 55212949148. 
variáveis, com uma cônica: elípse, hipérbole ou parábola; daí as conhecidas equações (operadores ou problemas) elípticas, hiperbólicas ou parabólicas (veja a parágrafo 1.1). Há também exemplos de equações de segundo ordem, como por exemplo a equação de Tricomi. $y u_{x x}+u_{y y}=0$, que é de tipo misto: é elíptica no semi-plano $y>0$ e é hiperbólica no semi-plano $y<0$.

Outra classificação bem sucedida é a de sistemas hiperbólicos de equações diferenciais parciais lineares de primeira ordem.

Tornou-se também hábito entre profissionais que lidam com EDP's denominar hiperbólico um problema de Cauchy cuja solução é obtida por propagação dos dados iniciais como no caso da equação da onda, parabólico um problema de Cauchy cuja solução torna-se regular num tempo infinitamente pequeño, independentemente da regularidade dos dados iniciais, como no caso da equação do calor elíptico um problema de valores de contorno cuja determinação da solução no interior da região considerada depende do seu conhecimento no contorno.

Generalizações no sentido de classificar EDP's de quaisquer ordens, tipos e números de variáveis independentes em elípticas, hiperbólicas e parabólicas foram feitas principalmente por Petrovsky [15], Courant-Hilbert [3], Friedricks [6], Garding [7], Leray [13], Agmon-Douglis-Nirenberg [1], Hormander [11] entre outros. Porém muitas EDP's ficam de fora dessas classificações.

Um dos nossos objetivos futuros é fazer uma análise que ligue a classificação usual em "tipos", de um tal sistema, segundo denominações que esses e outros autores vem empregando na literatura, com "teoremas de existência, unicidade e dependência contínua de soluções com respeito a dados, estabilidade e regularidade" de problemas de valores iniciais e de contorno, modelados por tais sistemas.

\subsection{Classificação de uma EDP de segunda ordem}

Uma equação semi-linear de segunda ordem em duas variáveis $x$ e $y$

$$
a_{11} u_{x x}+2 a_{12} u_{x y}+a_{22} u_{y y}+f\left(x, y, u, u_{x}, u_{y}\right)=0 \text {, }
$$

cujos:coeficientes $a_{i j}$ são funções contínuas sobre uma região $\Omega$ do plano pode ser transformada por meio de uma mudança de variáveis linear, respectivamente, numa das seguintes formas canônicas: $v_{\xi \xi}+v_{\eta \eta}+g\left(\xi, \eta, v, v_{\xi}, v_{\eta}\right)=0$, dita elíptica, $v_{\xi \xi}-v_{\eta \eta}+g\left(\xi, \eta, v, v_{j}, v_{\eta}\right)=0$, dita hiperbólica ou $v_{\xi \xi}+g\left(\xi, \eta, v, v_{\xi}, v_{\eta}\right)=0$, dita 
parabólica, em função do discriminante $\Delta=a_{11} a_{22}-a_{12}^{2}$ ser, respectivamente, positivo, negativo ou nulo.

No caso de $n$ variáveis

$$
\sum_{i, j=1}^{n} a_{i j} u_{x_{i} x_{j}}+f\left(x_{1}, \cdots, x_{n}, u, u_{x_{1}}, \cdots, u_{x_{n}}\right)=0
$$

onde $a_{i j}=a_{j i}(i, j=1, \cdots, n)$, tomando-se uma matriz quadrada $B$ de ordem $n$ que diagonaliza a matriz simétrica $A$, temos que a mudança de variáveis $\xi_{k}=\sum_{m=1}^{n} b_{m k} x_{m}$, $k=1, \cdots, n$ transforma esta equação em

$$
\sum_{k=1}^{n} d_{k} u_{\xi_{k} \xi_{k}}+g\left(\xi_{i}, u, u_{\xi_{j}}\right)=0
$$

sendo $B$ escolhida tal que os $d_{i}^{\prime} s$ são iguais a $1,-1$ ou 0 . Temos então a seguinte:

Definição 1.1 A equação (2) é dita elíptica se todos os $d_{i}^{\prime} s$ são iguais a 1 ou todos são iguais a-1. A EDP é dita hiperbólica se nenhum $d_{i}$ se anula, um único é igual a 1 e todos os demais são iguais a -1 ou vice-versa; se nenhum $d_{i}$ se anula, se ao menos dois dêles são negativos e ao menos dois dêles são positivos, a EDP é dita ultrahiperbólica. Se exatumente um dos dis é 0 e todos os demais têm o mesmo sinal, a EDP é dita parabólica.

Note que existem exemplos fora dessa definição, se $n \geq 4$.

\section{POLINÔMIO CARACTERÍSTICO DE UM SISTEMA DE EQUAÇÕES DIFERENCIAIS PARCIAIS}

Consideremos um sistema linear

$$
\left\{\begin{array}{c}
a_{11}(x . D) u_{1}+a_{12}(x, D) u_{2}+\cdots+a_{1 n}(x, D) u_{n}=f_{1}(x) \\
a_{21}(x . D) u_{1}+a_{22}(x, D) u_{2}+\cdots+a_{2 n}(x, D) u_{n}=f_{2}(x) \\
\vdots \\
a_{n 1}(x . D) u_{1}+a_{n 2}(x, D) u_{2}+\cdots+a_{n n}(x, D) u_{n}=f_{n}(x)
\end{array}\right.
$$


onde os $a_{i j}(x, D),(i, j=1, \cdots, n)$, são operadores diferenciais lineares polinomiais da forma

$$
a_{i j}(x, D)=\sum_{|\alpha| \leq m_{i j}} a_{i j \alpha}(x) D^{\alpha}
$$

$x=\left(x_{0}, x_{1}, \cdots, x_{p}\right) \in I R^{p+1}, m_{i j}$ é um inteiro não-negativo e onde a notação de multiíndice é usada:

$$
\begin{gathered}
D_{k}=\frac{\partial}{\partial x_{k}}(0 \leq k \leq n), D=\left(D_{0}, \cdots, D_{p}\right), D^{\alpha}=D_{0}^{\alpha_{0}} \cdots D_{p}^{\alpha_{p}}=\frac{\partial^{|\alpha|}}{\partial x_{0}^{\alpha_{0}} \cdots \partial x_{p}^{\alpha_{p}}} \\
\alpha=\left(\alpha_{0}, \cdots, \alpha_{p}\right) \in \mathbb{I N}^{p+1},|\alpha|=\alpha_{0}+\alpha_{1}+\cdots+\alpha_{p} .
\end{gathered}
$$

Por comodidade $a_{i j k}(x)$ e e $f_{i}(x)$ são funções contínuas reais ou complexas numa região de $I R^{n+1}$.

\subsection{Polinômio Característico no sentido de Birkhoff}

Para um sistema linear (3)(4), definimos como em [2], o polinômio característico como sendo $\chi(x, D)$ onde

$$
\chi(x, D)=\operatorname{det}\left(a_{i j}(x, D)\right) .
$$

Mais explícitamente. sendo $\xi=\left(\xi_{0}, \xi_{1}, \cdots, \xi_{p}\right)$ um vetor de coordenadas reais, temos

$$
\chi(x, \xi)=\operatorname{det}\left(a_{i j}(x, \xi)\right)
$$

onde com as notações usuais, para um multi-índice $\alpha, \xi^{\alpha}=\xi_{0}^{\alpha_{0}} \xi_{1}^{\alpha_{1}} \cdots \xi_{p}^{\alpha_{p}}$.

O polinômio $\chi_{H}(x, \xi)$ definido como a soma dos monômios de grau máximo $m$ de $\chi(x, \xi)$, é um polinômio homogêneo de grau $m$ em $\xi$.

\subsection{Polinômio Característico no sentido de Petrovsky}

Seja

$$
a_{i j .}^{p r}(x, D)=\sum_{|\alpha|=m_{j}} a_{i j \alpha}(x) D^{\alpha}
$$

onde para cada $j, m_{j}=\max _{i} m_{i j}$. Note que $a_{i j}^{p r}(x, D)=0$ se $m_{i j}<m_{j}$. O símbolo principal do sistema é o polinômio $P(x, D)$ definido por:

$$
P(x, D)=\operatorname{det}\left(a_{i j}^{p r}(x, D)\right)
$$




\subsection{Polinômio Característico no sentido de Agmon-Douglis-Nirenberg e Leray}

Escolhamos para o sistema (3)(4), como em [1] ou [13], uma coleção de inteiros $s_{1}, \cdots, s_{n}$ não positivos ( $s_{i}$ correspondente a linha $i, 1 \leq i \leq m$ do sistema) e $t_{1}, \cdots, t_{n}$ não negativos ( $t_{j}$ correspondente a coluna $j, 1 \leq j \leq n$ do sistema) tais que $m_{i j} \leq s_{i}+t_{j}$ e consideremos o polinômio associado ao sistema e a escolha $\left(s_{i}, t_{j}\right)$ :

$$
L(x, D)=\operatorname{det}\left(a_{i j}^{\left(s_{i}, t_{j}\right)}(x, D)\right)
$$

onde

$$
a_{i j}^{\left(s_{i}, l_{j}\right)}(x, D)=\sum_{|\alpha|=s_{i}+l_{j}} a_{i j \alpha}(x) D^{\alpha}
$$

e, convecionamos que $a_{i j}\left(s_{i}, t_{j}\right)=0 \mathrm{se}, s_{i}+t_{j}<0$.

Tem-se que $P(x, D)$ é um polinômio de grau $m_{1}, \cdots, m_{n}$, enquanto que $L(x, D)$ é um polinômio de grau $s_{1}+\cdots+s_{n}+t_{1}+\cdots+t_{n}$, pois cada parcela no desenvolvimento de (8) é da forma

$$
a_{1 j_{1}}^{\left(s_{1}, t_{j_{1}}\right)}(x, D) \cdots a_{n j_{n}}^{\left(s_{n}, i_{j_{n}}\right)}(x, D)
$$

de grau $s_{1}+t_{j_{1}}+\cdots+s_{n}+t_{j n}=s_{1}^{\prime}+\cdots+s_{n}+t_{1}+\cdots+t_{n}$ pois $\left\{j_{1}, \cdots, j_{n}\right\}=\{1, \cdots, n\}$.

Note que o sistema $\left(s_{i}-c, t_{j}+c\right)$ é equivalente ao sistema $\left(s_{i}, t_{j}\right)$ para qualquer inteiro $c$. Se $s_{1}=\cdots=s_{n}$ então $L(x, D)=P(x, D) \operatorname{com} t_{j}=m_{j}$ (ou $s_{1}^{\prime}=\cdots=s_{n}^{\prime}=0$, $\left.t_{j}^{\prime}=m_{j}-s_{i}\right)$.

\subsection{Polinômio Característico no sentido de Courant-Hilbert}

O polinômio característico de (3)(4) é definido como em [3] levando-se em conta apenas os coeficientes das derivadas de mais alta ordem $m=\max _{1 \leq i, j \leq n} m_{i j}$ :

$$
Q(x, D)=\operatorname{det}\left(\sum_{|\alpha|=m} a_{i j \alpha}(x) D^{\alpha}\right) .
$$

Em [3] considera-se. geralmente, sistemas onde cada equação possui a mesma ordem $m$, neste caso $Q$ é de grau $m n$.

\section{5 Ėxemplos de polinômios característicos}

Alem das tabelas 1 e 2 que mostram os polinômios característicos dos sistemas mais elementares, temos: 


\section{Sistema de Elasticidade Infinitesimal.}

$$
\left\{\begin{array}{l}
\mu \Delta u_{1}+(\lambda+\mu) \frac{\partial^{2} u_{1}}{\partial y_{1}^{2}}+(\lambda+\mu) \frac{\partial^{2} u_{2}}{\partial y_{1} \partial y_{2}}+(\lambda+\mu) \frac{\partial^{2} u_{3}}{\partial y_{1} \partial y_{2}}=0 \\
(\lambda+\mu) \frac{\partial^{2} u_{1}}{\partial y_{1} \partial y_{2}}+\mu \Delta u_{2}+(\lambda+\mu) \frac{\partial^{2} u_{2}}{\partial y_{2}^{2}+(\lambda+\mu) \frac{\partial^{2} u_{3}}{\partial y_{2} \partial y_{3}}=0} \\
(\lambda+\mu) \frac{\partial^{2} u_{1}}{\partial y_{1} \partial y_{3}}+(\lambda+\mu) \frac{\partial^{2} u_{2}}{\partial y_{2} \partial y_{3}}+\mu \Delta u_{3}+(\lambda+\mu) \frac{\partial^{2} u_{3}}{\partial y_{3}^{2}}=0
\end{array}\right.
$$

com $y=\left(y_{1}, y_{2}, y_{3}\right)$ em lugar de $\left(x_{0}, x_{1}, x_{2}\right), \quad D_{i}=\begin{gathered}\partial \\ \partial y_{i}\end{gathered}, \quad u_{j}=u_{j}(y)$ e $\Delta=D_{1}^{2}+D_{2}^{2}+D_{3}^{2}$, para $j=1,2,3$.

Temos para este sistema,

$$
\chi(D)=\chi_{11}(D)=P(D)=Q(D)=L(D)=\mu^{2}(\lambda+2 \mu) \Delta^{3} .
$$

Se p é um campo escalar, temos o sistema da Elasticidäde para um material incompressível

$$
\left\{\begin{array}{r}
A u-\nabla p=0 \\
\operatorname{div} u=0
\end{array}\right.
$$

onde A é o operador do primeiro membro de (9).

Usando as notações de (3) com $u_{4}=p$, temos

$$
\begin{gathered}
a_{i j}=\mu \delta_{i j} \Delta+(\lambda+\mu) D_{i} D_{j}, \quad 1 \leq i, j \leq 3 ; \\
a_{i 4}=\left(\delta_{4 i}-1\right) D_{i}, \quad a_{4 i}=\left(1-\delta_{4 i}\right) D_{i}, \quad i=1, \cdots, 4
\end{gathered}
$$

Então, neste caso

$$
P(D)=0
$$

e, com $s_{1}=s_{2}=s_{3}=-1, s_{4}=-2 ; t_{1}=t_{2}=t_{3}=3, t_{4}=2$, temos

$$
\chi(D)=L(D)=\operatorname{det}\left(\begin{array}{cccc}
\mu \Delta+\omega D_{1}^{2} & \omega D_{1} D_{2} & \omega D_{1} D_{3} & -D_{1} \\
\omega D_{1} D_{2} & \mu \Delta+\omega D_{2}^{2} & \omega D_{2} D_{3} & -D_{2} \\
\omega D_{1} D_{3} & \omega D_{2} D_{3} & \mu \Delta+\omega D_{3}^{2} & -D_{3} \\
D_{1} & D_{2} & D_{3} & 0
\end{array}\right)
$$

$=\mu^{2} \Delta^{3}, \omega=\lambda+\mu$. 
Para o sistema da Elasticidade para um material inextensível na direção do vetor $e_{1}=(1,0,0)$

$$
\left\{\begin{aligned}
A u-\frac{\partial p}{\partial y_{1}}(1,0,0) & =0 \\
\frac{\partial u_{1}}{\partial y_{1}} & =0
\end{aligned}\right.
$$

\begin{tabular}{|c|c|c|c|c|}
\hline SISTEMA & $\chi\left(D_{0}, D_{1}\right)$ & $\chi_{H}\left(D_{0}, D_{1}\right)$ & $P\left(D_{0}, D_{1}\right)$ & $L\left(D_{0}, D_{1}\right)$ \\
\hline$u_{\|}-u_{x x}=0$ & $D_{0}^{2}-D_{1}^{2}$ & $D_{0}^{2}-D_{1}^{2}$ & $D_{0}^{2}-D_{1}^{2}$ & $\begin{array}{l}D_{0}^{2}-D_{1}^{2} \\
s_{1}=0 \\
t_{1}=2\end{array}$ \\
\hline$u_{t}-u_{x x}=0$ & $D_{0}-D_{1}^{2}$ & $-D_{1}^{2}$ & $-D_{1}^{2}$ & $\begin{array}{l}-D_{1}^{2} \\
s_{1}=0 \\
t_{1}=2\end{array}$ \\
\hline$u_{t}+u_{x x}=0$ & $D_{0}+D_{1}^{2}$ & $D_{1}^{2}$ & $\begin{array}{l}D_{1}^{2} \\
\end{array}$ & $\begin{array}{l}D_{1}^{2} \\
s_{1}=0 \\
t_{1}=2\end{array}$ \\
\hline$u_{t \prime}+u_{x x}=0$ & $D_{0}^{2}+D_{1}^{2}$ & $D_{0}^{2}+D_{1}^{2}$ & $D_{0}^{2}+D_{1}^{2}$ & $\begin{array}{l}D_{0}^{2}+D_{1}^{2} \\
s_{1}=0 \\
t_{1}=2\end{array}$ \\
\hline$\left\{\begin{array}{c}u_{t}-v_{x}=0 \\
-u_{x}+v_{t}=0\end{array}\right.$ & $D_{0}^{2}-D_{1}^{2}$ & $D_{0}^{2}-D_{1}^{2}$ & $D_{0}^{2}-D_{1}^{2}$ & $\begin{array}{l}D_{0}^{2}-D_{1}^{2} \\
s_{1}=s_{2}=0 \\
t_{1}=t_{2}=1\end{array}$ \\
\hline$\left\{\begin{array}{l}u_{t}-v_{x}=0 \\
u_{x}+v_{t}=0\end{array}\right.$ & $D_{0}^{2}+D_{1}^{2}$ & $D_{0}^{2}+D_{1}^{2}$ & $D_{0}^{2}+D_{1}^{2}$ & $\begin{array}{l}D_{0}^{2}+D_{1}^{2} \\
s_{1}=s_{2}=0 \\
t_{1}=t_{2}=1\end{array}$ \\
\hline$\left\{\begin{array}{l}-u_{x}+v_{t}=0 \\
-u+v_{x}=0\end{array}\right.$ & $D_{0}-D_{1}^{2}$ & $-D_{1}^{2}$ & $-D_{1}^{2}$ & $\begin{array}{l}-D_{1}^{2} \\
s_{1}=s_{2}=0 \\
t_{1}=t_{2}=1\end{array}$ \\
\hline
\end{tabular}

Table 1: Polinômios característicos nos exemplos usuais. 


\begin{tabular}{ll}
\hline SISTEMA & $\chi\left(D_{0}, D_{1}\right)$ \\
\hline
\end{tabular}

Euler-Bernoulli

$$
\begin{array}{cc}
u_{t \prime}(x, t)+c^{2} u_{x x x x}(x, t)=0 & D_{0}^{2}+c^{2} D_{1}^{4} \\
\text { Vlasov } & \\
u_{t t}(x, t)+c^{2} u_{x x x x}(x, t)-r^{2} u_{t x x}(x, t)=0 & D_{0}^{2}+c^{2} D_{i}^{4}-r^{2} D_{0} D_{1}^{2} \\
\text { Timoshenko } & \\
\begin{cases}u_{t \prime}(x, t)+\alpha^{2} \psi_{x}(x, t)-\alpha^{2} u_{x x}(x, t)=0 & D_{0}^{4}+\alpha^{2} \beta^{2} D_{1}^{4} \\
\psi_{t \prime}(x, t)+\left(\alpha^{2} / r^{2}\right) \psi(x . t) & +\left[\alpha^{2} / r^{2}-\left(\alpha^{2}+\beta^{2}\right) D_{1}^{2}\right] D_{0}^{2} \\
-\left(\alpha^{2} / r^{2}\right) u_{x}(x, t)-\beta^{2} \psi_{x x}(x, t)=0 & \end{cases}
\end{array}
$$

Table 2: Equações da teoria linear clássica de vibrações transversais de vigas.

temos $P(D)=0$, enquanto que, com $s_{1}=s_{2}=s_{3}=-1, \quad s_{4}^{*}=-2 ; t_{1}=t_{2}=t_{3}=3$, $t_{4}=2$, temos

$$
\begin{aligned}
& \chi(D)=L(D)=\operatorname{det}\left(\begin{array}{cccc}
\mu \Delta+\omega D_{1}^{2} & \omega D_{1} D_{2} & \omega D_{1} D_{3} & -D_{1} \\
\omega D_{1} D_{2} & \mu \Delta+\omega D_{2}^{2} & \omega D_{2} D_{3} & 0 \\
\omega D_{1} D_{3} & \omega D_{2} D_{3} & \mu \Delta+\omega D_{3}^{2} & 0 \\
D_{1} & 0 & 0 & 0
\end{array}\right) \\
& =\mu D_{1}^{2}\left(\mu \Delta^{2}+\omega \Delta\left(D_{2}^{2}+D_{3}^{2}\right)\right), \omega=\lambda+\mu .
\end{aligned}
$$

\section{SISTEMAS ELÍPTICOS NO SENTIDO DE AGMON-DOUGLIS-NIRENBERG,} COURANT-HILBERT E PETROVSKY

Considere o sistema (3)(4) e $P(x, D)$ o polnômico de (6) para um $x$ fixado.

Definição 3.1 O sistema (3)(4) é elíptico

i. no sentido de Petrovsky no ponto $x$ se, para todo $\xi=\left(\xi_{0}, \xi_{1}, \cdots, \xi_{p}\right) \in \mathbb{R}^{p+1}$,

$$
P(x, \xi)=0 \Leftrightarrow \xi=0 .
$$

ii. no sentido de Agmon-Douglis-Nirenberg no ponto $x$ se, o polinômio $L(x, D)$ de (8) é tal que paru todo $\xi=\left(\xi_{0}, \xi_{1}, \cdots, \xi_{p}\right) \in \mathbb{R}^{p+1}$,

$$
L(x, \xi)=0 \Leftrightarrow \xi=0 .
$$


iii. no sentido de Courant-Hilbert no ponto $x$ se, para todo $\xi=\left(\xi_{0}, \xi_{1}, \cdots, \xi_{p}\right) \in \mathbb{R}^{p+1}$,

$$
Q(x, \xi)=0 \Leftrightarrow \xi=0 .
$$

\subsection{Exemplos de sistemas elípticos}

A equação $u_{l \prime}+u_{x x}=0$ tem

$$
P\left(\xi_{0}, \xi_{1}\right)=L\left(\xi_{0}, \xi_{1}\right)=\xi_{0}^{2}+\xi_{1}^{2}=0 \Leftrightarrow\left(\xi_{0}, \xi_{1}\right)=0
$$

e portanto ela é elíptica no sentido de Petrovsky.

Transformando essa equação no sistema

$$
\left\{\begin{array}{l}
u_{t}-v_{x}=0 \\
u_{x}+v_{t}=0
\end{array}\right.
$$

vemos também que $P(x, D)=L(x, D)=D_{0}^{2}+D_{1}^{2}$ e assim, $P(\xi)=L(\xi)=0 \Leftrightarrow \xi=0$.

Se essa mesma equação é transformada em

$$
\left\{\begin{array}{l}
\left(u_{1}\right)_{t}+\left(u_{2}\right)_{x}=0 \\
u_{t}-u_{1}-u_{2}=0 \\
u_{x}-u^{2}-u^{2}
\end{array}\right.
$$

tem $P(x, D)=0$ e portanto não é elíptico no sentido de Petrovsky, embora, com $s_{1}=0, s_{2}=s_{3}=-1 ; t_{1}=2, t_{2}=t_{3}=1$, temos $L(x, D)=D_{0}^{2}+D_{1}^{2}$ e portanto é elíptico no sentido de Agmon-Douglis-Nirenberg.

De (10), vemos que o sistema correspondente (9) é elíptico (em todos os sentidos acima definidos); de (12)(15), vemos que o exemplo (11) é elíptico no sentido de Agmon-Douglis-Nirenberg porém não o é no sentido de Petrovsky. Os demais exemplos não são elípticos.

Note que elipticidade no sentido de Courant-Hilbert implica elipticidade no sentido de Petrovsky que por sua vez, implica elipticidade no sentido de Agmon-DouglisNirenberg.

\section{SISTEMAS HIPERBÓLICOS}

Consideremos um sistema de $n$ equações em $n$ incógnitas, do tipo

$$
\left\{\begin{array}{c}
D_{01}^{M_{1} u_{1}} u_{1}=a_{11}(x, D) u_{1}+\cdots+a_{1 n}(x, D) u_{n} \\
\vdots \\
D_{0}^{M_{n}} u_{n}=a_{n 1}(x, D) u_{1}+\cdots+a_{n n}(x, D) u_{n}
\end{array}\right.
$$


onde $x=\left(x_{0}, \cdots, x_{p}\right) \quad D=\left(D_{0}, D_{1}, \cdots, D_{p}\right)$ e para $1 \leq i, j \leq n, M_{i}$ inteiros $\geq 1$

$$
a_{i j}(x, D)=\sum_{|\alpha|=M_{i}, \alpha_{0}<M_{i}} a_{i j \alpha}(x) D^{\alpha}
$$

No caso $M_{1}=\cdots=M_{n}=1$, o sistema é da forma

$$
\left\{\begin{array}{c}
D_{0} u_{1}=a_{11}(x, D) u_{1}+\cdots+a_{1 n}(x, D) u_{n} \\
\vdots \\
D_{0} u_{n}=a_{n 1}(x, D) u_{1}+\cdots+a_{n n}(x, D) u_{n}
\end{array}\right.
$$

sendo $a_{i j}(x, D)=\sum_{1 \leq k \leq p} a_{i j k}(x) D_{k}$.

Temos os polinômios: $\left(\operatorname{com} M=M_{1}+\cdots+M_{n}\right)$

$$
\chi(x, D)=\operatorname{det}\left(\begin{array}{cccc}
D_{0}^{M_{1}}-a_{11}(x, D) & a_{12}(x, D) & \cdots & a_{1 n}(x, D) \\
\cdots & \cdots & \cdots & \cdots \\
a_{n 1}(x, D) & a_{n 2}(x, D) & \cdots & D_{0}^{M_{n}}-a_{n n}(x, D)
\end{array}\right)
$$

sendo $l(x, D)$, como polinômio em $D$ de grau $<M$ em relação a $D_{0}$.

Consideremos, como exemplo (com $p=1, M_{1}=1, M_{2}=2, m_{1}=m_{2}=2$ ), o sistema:

$$
\left\{\begin{array}{l}
D_{0} u_{1}=a_{11}(x) D_{1} u_{1}+a_{12} D_{1} u_{2} \\
D_{0}^{2} u_{2}=a_{21}(x) D_{1}^{2} u_{1}+a_{22} D_{0} D_{1} u_{2}
\end{array}\right.
$$

ou,

$$
\left\{\begin{array}{l}
D_{0} u_{1}-a_{11}(x) D_{1} u_{1}-a_{12} D_{1} u_{2}=0 \\
D_{0}^{2} u_{2}-a_{21}(x) D_{1}^{2} u_{1}-a_{22} D_{0} D_{1} u_{2}=0
\end{array}\right.
$$

para o qual,

$$
P(x, D)=\operatorname{det}\left(\begin{array}{cc}
0 & 0 \\
-a_{21}(x) D_{1}^{2} & D_{0}^{2}-a_{22}(x) D_{0} D_{1}
\end{array}\right) \equiv 0
$$

Por outro lado, escolhendo-se $t_{1}=t_{2}=\cdots=t_{n}=m=\operatorname{máx}\left\{M_{i}: 1 \leq i \leq n \quad\right.$ e, $s_{i}=M_{i}-m$, temos que $s_{i}+t_{j}=\left(M_{i}-m\right)+m=M_{i}$, portanto

$$
L(x, D)=\chi(x, D) \text {. }
$$

Em [14, página 530] temos a seguinte definição 
Definição 4.1 O sistema (17)(18) é denominado hiperbólico no sentido de Petrovsky se a equação em $\xi_{0}$.

$$
\chi(x, \xi)=\xi_{0}^{M}+l\left(x, \xi_{0}, \xi_{1}, \cdots, \xi_{p}\right)=0
$$

possui, para cada $\left(\xi_{1}, \cdots, \xi_{p}\right) \neq 0$, apenas raizes reais e distintas.

A equação $u_{\|}=u_{x_{1} x_{1}}$ é hiperbólica no sentido de Petrovsky pois, neste caso, para cada $\xi_{1} \neq 0$,

$$
L\left(t, x_{1}, \xi_{0}, \xi_{1}\right)=\xi_{0}^{2}-\xi_{1}^{2}=0
$$

possui raízes $\xi_{0}= \pm\left|\xi_{1}\right|$.

Vemos que para um sistema (17)(18) ou, mais geralmente, com (18) substituída por:

$$
a_{i j}(x, D)=\sum_{|\alpha| \leq M_{i}, \alpha_{0}<M_{i}} a_{i j \alpha}(x) D^{\alpha} .
$$

a definição de hiperbolicidade de Petrovsky leva em consideração o mesmo polinômio usado por Agmon-Douglid-Nirenberg e Leray.

De [3, páginas 580 e 590] e [2], temos a seguinte

Definição 4.2 Um sistema (3)(4) é dito totalmente hiperbólico no sentido de CourantHilbert ou no sentido de Petrovsky em um ponto $x$ se, dado $\left(\xi_{1}, \cdots, \xi_{p}\right) \neq 0$ qualquer, a equação em $\xi_{0}$, (se necessário com uma mudança de coordenadas conveniente):

$$
Q\left(x, \xi_{0}, \xi_{1}, \cdots, \xi_{p}\right)=0
$$

Possui apenas raízes reais distintas, ou seja, sendo $Q(x, \xi)=0$ de grau $\dot{m}^{\prime}$ em $\xi_{0}$, ela possui $m^{\prime}$ raizes recis distintas.

Em [3], é geralmente considerado que cada equação do sistema possui a mesma ordem $m \mathrm{e}, m^{\prime}=m n$.

De [3], temos

\section{Definição 4.3}

i. Um sistema (19) é dito totalmente hiperbólico no sentido de Courant-Hilbert em um ponto $x$ se, pura cada $\left(\xi_{1}, \cdots, \xi_{p}\right) \neq 0$, a equação em $\xi_{0}$ :

$$
\chi_{H}\left(x, \xi_{0}, \xi_{1}, \cdots, \xi_{p}\right)=0,
$$

possui apenas rcizes reais, não necessáriamente distintas. 
ii. Um sistema (19) é dito hiperbólico no sentido de Garding em um ponto $x$ se, para cada $q \in \mathbb{R}^{p}$, todas as raízes $\lambda_{k}(q)$ da equação $\chi(\lambda, i q)=0$, com relação à $\lambda$, satisfazem:

$$
\operatorname{Re}\left(\lambda_{k}(q)\right) \leq C .
$$

Reforçamos que a constante $C$ não debe depender de $k=1, \cdots, n$ e de $q \in \mathbb{R}^{p}$.

\subsection{Exemplos de sistemas hiperbólicos}

Voltemos as equações de deflexões de vigas. Da da tabela 2, temos para a equação de Euler-Bernoulli, $\chi\left(D_{0}, D_{1}\right)=D_{0}^{2}+c^{2} D_{1}^{4}$, para a equação de Vlasov,

$$
\chi\left(D_{0}, D_{1}\right)=D_{0}^{2}+c^{2} D_{1}^{4}-r^{2} D_{0} D_{1}^{2}
$$

e para o sistema de Timoshenko,

$$
\chi\left(D_{0}, D_{1}\right)=D_{0}^{4}+\alpha^{2} \beta^{2} D_{1}^{4}+\left[\alpha^{2} / r^{2}-\left(\alpha^{2}+\beta^{2}\right) D_{1}^{2}\right] D_{0}^{2}
$$

Então, a equação de Vlasov e o sistema de Timoshenko são hiperbólicos no sentido de Petrovsky se, $r^{2}>4 a^{2}$ e $\alpha \neq \beta$, respectivamente. Todos os três exemplos são hiperbólicos no sentido de Garding se extendemos sua definição para sistema com segundo membro de ordem maior que um.

\section{SISTEMAS PARABÓLICOS}

Considere o sistema

$$
D_{0}^{M_{i}} u_{i}=\sum_{j=1}^{n} a_{i j}(x, D) u_{j}, \quad i=1, \cdots, n
$$

onde

$$
a_{i j}(x, D)=\sum_{\alpha} a_{i j \alpha}(x) D^{\alpha}
$$

Os índices $\alpha$ de (21) são considerados por Petrovsky [15], páginas 404-405, possuindo um peso $b$ para as derivações $D_{0}$, ou seja, a cada fator $D_{0}$ corresponde os fatores $D_{1}^{b}, \cdots, D_{p}^{b}$. Assim, ele considera (20)(21), onde em (21) os multi-índices $\alpha$ satisfazem

$$
\alpha_{0} b+\alpha_{1}+\cdots+\alpha_{p} \leq M_{i} b, \quad b \alpha_{0}<M_{i}, \quad 1 \leq i \leq n
$$

\subsection{Exemplos de sistemas parabólicos}

a) A equação $u_{x 0}=u_{x \mid x 1}$ é caso particular com $n=1, \quad b=2, \quad M_{1}=1$ $\left(2 \alpha_{0}+\alpha_{1} \leq 2 \Rightarrow \alpha_{0}=0\right), \log 0$

$$
D_{0} u=a_{1(0,0)}(x) u+a_{2(0,1)}(x) D_{1} u+a_{3(0,2)}(x) D_{1}^{2} u,
$$

onde $a_{1(0,0)}=a_{2(1), 1)}=0, a_{3(0,2)}=1$. 
b) A equação $u_{x 0}=-u_{x 1}-u_{x 1 x 1 x 1}$, corresponde ao caso em que $n=1, M_{1}=1, b=3$,

$$
D_{0} u=a_{1(0,0)}(x) u+a_{2(0,1)}(x) D_{1} u+a_{3(0,2)}(x) D_{1}^{2} u+a_{4(0,3)}(x) D_{1}^{3} u,
$$

$3 \alpha_{0}+\alpha_{1} \leq 3 \Rightarrow \alpha_{0}=0$.

Neste exemplo, $a_{1(0,0)}=0, a_{2(0,1)}=-1, a_{3(0,2)}=0, a_{4(0,3)}=-1$.

Definição 5.1 O sistema (20)-(22), é dito parabólico no sentido de Petrovsky no ponto $x$ se, para cada $\left(\xi_{1}, \cdots, \xi_{p}\right) \in \mathbb{R}^{p}, \xi_{1}^{2}+\cdots+\xi_{p}^{2}=1$, as raízes $\xi_{0}=\xi_{0}(x, \xi)$ da equação em $\xi_{0}$

$$
\chi^{0}\left(x, \xi_{0}, i \xi_{1}, \cdots, i \xi_{p}\right)=0,
$$

são tais que $\operatorname{Re}\left(\xi_{0}(x . \xi)\right)<-\delta(x)$, onde $\delta(x)$ é uma função positiva.

$$
\begin{gathered}
\text { Aquí, com } \xi=\left(\xi_{0}, \xi_{1}, \cdots, \xi_{p}\right) \\
\chi^{0}\left(x, \xi_{0}, \xi_{1}, \cdots, \xi_{p}\right)=\operatorname{det}\left(\begin{array}{cccc}
\xi_{0}^{M_{1}}-a_{11}^{0}(x, \xi) & a_{12}^{0}(x, \xi) & \cdots & a_{1 n}^{0}(x, \xi) \\
\cdots & \cdots & \cdots & \cdots \\
a_{n 1}^{0}(x, \xi) & a_{n 2}^{0}(x, \xi) & \cdots & \xi_{0}^{M_{n}}-a_{n n}^{0}(x, \xi)
\end{array}\right) \equiv 0,
\end{gathered}
$$

onde

$$
a_{i j}^{0}(x, \xi)=\sum_{\alpha_{0} b+\alpha_{1}+\cdots+\alpha_{p}=M_{i} b, b \alpha_{0}<M_{i}} a_{i j \alpha}(x) \xi^{\alpha}
$$

Se existe una constante $\delta>0$ satisfazendo para todo $\left(\xi_{1}, \cdots, \xi_{p}\right) \in \mathbb{R}^{p}$, $\xi_{1}^{2}+\cdots+\xi_{p}^{2}=1, \operatorname{Re}\left(\xi_{0}(x, \xi)\right)<-\delta(x) ;$ o sistema (20)-(22) é dito uniformemente parabólico no sentido de Petrovsky.

Lema 5.1 $\forall k \neq 0, \xi_{0}\left(k \xi_{1}, \cdots, k \xi_{p}\right)=k^{b} \xi_{0}\left(k \xi_{1}, \cdots, k \xi_{p}\right)$.

Comentário 5.1 Se (20)-(22), é parabólico, no sentido de Petrovsky, então b é par, pois do contrário, com $k=-1$ teríamos alguma raiz $\xi_{0}$ com parte real positiva. De fato, sendo $\operatorname{Re}\left(\xi_{0}\left(\xi_{1} \cdots, \xi_{p}\right)\right)<0$ teríamos $\operatorname{Re}\left(\xi_{0}\left(-\xi_{1}, \cdots,-\xi_{p}\right)\right)>0$.

Para quaisquer $\eta=\left(\xi_{1}, \cdots, \xi_{p}\right) \neq 0$, tem-se $\|\eta\|^{-b} \xi_{0}(\eta)=\xi_{0}\left(\frac{\eta}{\|\eta\|}\right)$, se $\operatorname{Re}\left(\xi_{0}\left(\frac{\eta}{\|\eta\|}\right)\right)<-\delta$, então

$$
\forall \eta \neq 0, \operatorname{Re}\left(\xi_{0}(\eta)\right)<-\delta\|\eta\|^{b}
$$

Nos exemplos anteriores temos 
- $u_{t}=u_{x x}$ parabólica no sentido de Petrovsky pois

$$
\chi^{0}\left(\xi_{0}, \xi_{1}\right)=\xi_{0}+\xi_{1}^{2}=0 \Leftrightarrow \xi_{0}=-\xi_{1}^{2}=-1
$$

- $u_{t}=-u_{x}-u_{x x x}$ temos $b=3 ; \chi^{0}\left(\xi_{0}, \xi_{1}\right)=\xi_{0}-i \xi_{1}^{3}=0 \Leftrightarrow \xi_{0}= \pm i$, e portanto não é parabólica no sentido de Petrovsky.

Prova do lema 5.1: Consideremos que os multi-índices $\alpha$ satisfazem as relações definindo $a_{i j}^{0}$ :

$$
b \alpha_{0}+\alpha_{1}+\cdots+\alpha_{p}=b M_{i}, \quad b \alpha_{0}<M_{i}, \quad 1 \leq i \leq n
$$

Mostramos que

$$
\begin{aligned}
\chi^{0}\left(x, \xi_{0}, i k \xi_{1}, \cdots, i k \xi_{1}\right) & =\operatorname{det}\left(\xi_{0}^{M_{1}} \delta_{i j}-a_{i j}^{0}\left(x, \xi_{0}, i k \xi_{1}, \cdots, i k \xi_{p}\right)\right) \\
& =\operatorname{det}\left(k^{b M_{i}}\left[\left(k^{-b} \xi_{0}\right)^{M_{i}}-a_{i j}^{0}\left(x, k^{-b} \xi_{0}, i \xi_{1}, \cdots, i \xi_{p}\right)\right]\right) \\
& =k^{\left(M_{1}+\cdots+M_{N}\right) b} \chi^{0}\left(x, k^{-b} \xi_{0}, i \xi_{1}, \cdots, i \xi_{p}\right)
\end{aligned}
$$

Assim,

$\xi_{0}$ é raiz de $q(\lambda)=\chi^{0}\left(x, \xi_{0}, i k \xi_{1}, \cdots, i k \xi_{p}\right)=0 \Leftrightarrow k^{-b} \xi_{0}$ é raiz de $q(\lambda)=0$ ou seja,

$k^{-b} \xi_{0}\left(x, k \xi_{1}, \cdots, k \xi_{p}\right)=\xi_{0}\left(x, k \xi_{1}, \cdots, k \xi_{p}\right)$ ou ainda

$\xi_{0}\left(x, k \xi_{1}, \cdots, k \xi_{p}\right)=k^{\prime \prime} \xi_{0}\left(x, \xi_{1}, \cdots, \xi_{p}\right)$

Para provar (25) temos de (23) e (24), que

$$
\begin{gathered}
\xi_{0}^{M_{1}} \delta_{i j}-a_{i j}^{0}\left(x, \xi_{0}, i k \xi_{1}, \cdots, i k \xi_{p}\right) \\
=\xi_{0}^{M_{1}} \delta_{i j}-\sum_{\alpha} a_{i j \alpha} \xi_{0}^{\alpha_{0}}\left(i k \xi_{1}\right)^{\alpha_{1}} \cdots\left(i k \xi_{p}\right) \alpha^{\alpha_{p}} \\
=\xi_{0}^{M_{1}} \delta_{i j}-\sum_{\alpha} a_{i j \alpha} \xi_{0}^{\alpha_{0}} k^{\alpha_{1}+\cdots+\alpha_{p}}\left(i \xi_{1}\right)^{\alpha_{1}} \cdots\left(i \xi_{p}\right)^{\alpha_{p}}
\end{gathered}
$$

(de (24), $\left.\alpha_{1}+\cdots+\alpha_{1}=b M_{1}-b \alpha_{0}\right)$

$$
\begin{gathered}
=\xi_{0}^{M_{1}} \delta_{i j} k^{b M_{i}} k^{-b M_{i}}-\sum_{\alpha} a_{i j \alpha} \xi_{0}^{\alpha_{0}} k^{b M_{i}} k^{-b \alpha_{0}}\left(i \xi_{1}\right)^{\alpha_{1}} \cdots\left(i \xi_{p}\right)^{\alpha_{p}} \\
=k^{h \Lambda I_{i}}\left\{\left(k^{-b} \xi_{0}\right)^{M_{i}} \delta_{i j}-\sum_{\alpha} a_{i j \alpha}\left(k^{-b} \xi_{0}\right)^{\alpha_{0}}\left(i \xi_{1}\right)^{\alpha_{1}} \cdots\left(i \xi_{p}\right)^{\alpha_{p}}\right\} \\
=k^{b M_{i}}\left\{\left(k^{-b} \xi_{0}\right)^{M_{i}} \delta_{i j}-a_{i j}^{0}\left(x, k^{-b} \xi_{0}, i \xi_{1}, \cdots, i \xi_{p}\right)\right.
\end{gathered}
$$

O restante da prova, segue-se de propriedades da função determinante 


\section{REGULARIDADE E HIPERBOLICIDADE}

Consideremos um sistema (3)(4)

$$
\begin{gathered}
\sum_{j=1}^{n} a_{i j}(D) v_{j}=0, i=1, \cdots, n \\
a_{i j}(D)=\sum_{|\alpha| \leq m_{i j}} a_{i j \alpha} D^{\alpha}
\end{gathered}
$$

com coeficientes $a_{i j \alpha}$ constantes reais.

Consideremos também o caso particular

$$
D_{0} u_{i}=\sum_{j=1}^{n} b_{i j}\left(D_{1}, \cdots, D_{p}\right) u_{j}, \quad i=1, \cdots, n
$$

$\operatorname{com} b_{i j}\left(D_{1}, \cdots, D_{p}\right)=\sum_{|\alpha| \leq m_{i j}} b_{i j \beta} D_{1}^{\beta_{1}} \cdots D_{p}^{\beta_{p}}$ onde não ocorre $D_{0}$. Para este caso, seja o polinômio característico em $\lambda$,

$$
\sigma(\lambda, \omega)=\operatorname{det}\left(\lambda I-\left(b_{k l}(i \omega)\right)_{1 \leq k, I \leq n}\right) .
$$

Definição 6.1 O sistema (28) é denominado

- estável se para todo $\omega$ real, todas as $n$ raízes $\lambda_{k}(\omega)$ de $\sigma(\lambda, \omega)=0$ são tais que $\operatorname{Re}\left(\lambda_{k}(\omega)\right)<0$

- regular se existe uma constante real $M$ tal que para todo $\omega, \operatorname{Re}\left(\lambda_{k}(\omega)\right)<M$, $k=1, \cdots, n$.

Observação 6.1 Da definição de polinômio característico $\chi(x, D)$ em (5), para o sistema (28), temos $\sigma(\lambda, \omega)=\chi(\lambda, i \omega)$. No caso em que $\chi=\chi_{H}$, sua parte principal de maior grau, então $\chi_{H}$ é homogêneo, digamos de grau m. Assim,

$$
\chi_{H}(\alpha \lambda, \alpha \omega)=\alpha^{m} \chi(\lambda, \omega) .
$$

e se $\lambda$ é raiz de $\chi_{11}(\lambda, \omega)=0$ vemos que $i \lambda$ é raiz de $\chi_{H}(\lambda, i \omega)=\sigma_{H}(\lambda, \omega)=0$. Portanto, todas as raizes de $\sigma_{H}(\lambda, \omega)=0$ têm partes reais nulas se e somente se $\chi_{H}(\lambda, \omega)=0$ possui todas as suas raizes reais.

Ainda da homogeneidade de $\chi_{H}(\lambda, \omega)$, segue-se que todas as raizes de $\sigma_{H}(\hbar, \omega)=0$ possuem parte real limitada, independentemente de $\omega$ se, e somente se forem imaginárias puras, ou seja, neste caso, o sistema é regular se e somente se, todas as raízes de $\sigma_{\|}(\lambda, \omega)=0$ são imaginárias puras, ou equivalentemente, se todas as raizes de $\chi_{H}(\lambda, \omega)=0$ são reais. 
O sistema (28) é regular se, e somete se, o polinômio $\sigma(\lambda, \omega-K)$ é estável, para algum $K>0$.

Nota 6.1 Da observação 6.1 vemos que no caso em que $\chi=\chi_{H}$, o sistema (26)(27) é regular se e somente se, todas as raizes de $\chi_{H}(\lambda, \omega)=0$ são reais. (Equivalência com a definição de sistema hiperbólico de Courant-Hilbert, (conforme [2])). Se tem também que o sistema é hiperbólico no sentido de Petrovsky (conforme [2]) se é regular e além disso $\chi_{H}(\lambda, \omega)=0$ possui para cada $\omega \neq 0$ todas as raizes reais $e$ distintas.

Analisemos agora o caso não homogêneo: $\chi \neq \chi_{H}$.

\section{Condição necessária para a regularidade:}

Lema 6.1 Se para cuda $\omega \neq 0$, as raízes de $\chi_{H}(\lambda, \omega)=0$ são reais distintas, então o sistema (26)(27) é regular (independentemente dos termos de ordens mais baixas de $\chi$ ). Nestas condições, dizemos que o sistema (26)(27) é completamente regular.

Observação 6.2 O sistema (26)(27) é hiperbólico no sentido de Courant-Hilbert se é regular (lema 6.1) e. reciprocamente, assim o será se é hiperbólico no sentido de Courant-Hilbert e $\chi_{11}(\lambda, \omega)=0$ possui para cada $\omega \neq 0$, raizes distintas (lema 6.1).

Finalmente notemos que se $\chi_{H}(\lambda, \omega)=0$ possuir raízes múltiplas, o sistema (26)(27) não é necessáriamente regular. Por exemplo, temos para $u_{\|}(t, x)=0$; $\chi(\lambda, \omega)=\lambda^{2}$ possui raízes reais multiplas e é regular pois $\chi=\chi_{H}$, porém, $u_{\|}+u_{x}=0$ não é regular pois $\chi_{H}(\lambda, i \omega)=\lambda^{2}$ possui raízes reais múltiplas e,

$$
\sigma(\lambda, i \omega)=\chi(\lambda, \omega)=\lambda^{2}+i \omega=0
$$

possui raízes por exemplo, para $\omega=-r^{2}, r>0$;

$$
\lambda_{1}(\omega)=\frac{r}{\sqrt{2}}(1+i):=-\lambda_{2}(\omega) ; \operatorname{Re}\left(\lambda_{k}(\omega)\right)=\frac{r}{\sqrt{2}} \rightarrow \infty \text { quando } \omega \rightarrow \infty
$$

\section{UM PROBLEMA SEM CLASSIFICAÇÃO}

Os resultados que exporemos a seguir podem ser encontrados em [9] ou [4].

O sistema da Elasticidade linear inextensivel na direção $e_{3}=(0,0,1)$ para um material homogéneo e isotrópico é dado por 


$$
\left\{\begin{aligned}
\mu \Delta u_{i}+(\lambda+\mu) \frac{\partial}{\partial x_{i}}(\operatorname{div} u)+\phi_{x_{3}} \delta_{i, 3} & =0 \\
\partial u_{3} & =0 \\
\partial x_{i} &
\end{aligned}\right.
$$

onde $\phi$ é uma função continuamente diferenciável arbitrária e $\delta_{i j}$ é o delta de Kronecker.

Análogamente ao sistema (16) onde a direção de inextensibilidade é $e_{1}=(1,0,0)$ temos para este caso

$$
P(D)=0 \text { e } \chi(D)=-\mu D_{3}^{2}\left(\mu \Delta^{4}+(\lambda+\mu) \Delta\left(D_{1}^{2}+D_{2}^{2}\right)\right) .
$$

Da segunda equação, temos que $u=u\left(x_{1}, x_{2}\right)$ na região $R$ considerada $\mathrm{e}$ conhecimento de $u_{3}$ em um ponto $P=\left(x_{1}{ }^{*}, x_{2}^{*}, x_{3}^{*}\right)$ da fronteira $\partial R$, implica que $u_{3}$ é conhecida em todo ponto da reta $x_{1}=x_{1}^{*}, x_{2}=x_{2}^{*}$. Portanto, se $u_{3}$ é dada num ponto $P$ da fronteira, ela não pode ser dada arbitrariamente em oütros pontos da reta $x_{1}=x_{1}^{*}$, $x_{2}=x_{2}^{*}$ sobre a fronteira, sob pena de a solução do problema não existir.

Então para a colocação do problema, definimos parte admissível da fronteira $\partial R$ : Por cada ponto $P$ de $\partial R$ trace um segmento de reta paralelo ao eixo $x_{3}$ (incluído em $\partial R$ ou dentro de R). () conjunto dos pontos (excetuando-se o próprio P) sobre este segmento de reta e simultanemeamente sobre $\partial R$ é denominado dual de $P$ e é denotado por $D(P)$.

Definição 7.1 Uma parte $A$ de $\partial R$ é dita admissível na direção $x_{3}$ se:

i. $\forall P \in A, D(P) \cap A=\phi ;$

ii. $\quad D(P) \cup A=\partial R$

O problema do deslocamento, o qual tem solução única se $\mu(\lambda+\mu)>0$ em $R$, conforme [9, Teorema 2], é o seguinte:

Problema (P1): Achar $u$ e $\phi$ satisfazendo (29), sendo dados

$$
\left\{\begin{array}{l}
u_{1}, u_{2} \text { dados em } \partial R ; \\
u_{;} \text {sobre uma parte admissível } A \text { na direção } x_{3}
\end{array}\right. \text {. }
$$

Observamos que este problema não é do tipo Dirichlet, nem Neuman e nem misto. 


\section{REFERENCES}

1. S. AGMON, A. DOUGLIS, L. NIRENBERG, Estimates Near the Boundary for Solutions of Elliptic Partial Differential Equations. Boundary Conditions, II, Comm. P. Appl. Math., vol XVII, 35-92, (1964).

2. G. BIRKHOFF, Classification of Partial Differential Equations, J.SIAM Appl. Math., vol. 2 (1), 57-67, (1954).

3. R. COURANT, D. HILbeRT, Methods of Mathematical Physics, vol. 2, Interscience Publishers, (1962).

4. M. J. H. DANTAS, Sobre Problemas de Contorno para Materiais Elásticos Anisotrópicos com Vínculo, $38^{\circ}$ Seminário Brasileiro de Análise, pp 49-73, (1993).

5. S. D. EIDELMAN, Paraholic System, Nirth-Holland, (1969).

6. K. O. FRIEDRICHS, Symmetric Positive Linear Differential Equations, Comm. P. Appl. Math., vol. XI, 333-418, (1958).

7. P. GARABEDIAN, Partial Differential Equations, Wiley, (1964).

8. L. GARDING, Linear Hyperbolic Differential Equations with Constant Coeficients, Acta Math., 85, 1$62,(1951)$.

9. M. HAYES, C. O. HORGAN, On the displacement boundary-value problem for inextensible elastic materials, Q. Jl. Mech. Appl. Match., Vol. XXVII, Pt. 3, (1975).

10. G. HELLWING, Partial Differential Equations, New York, Blaisdell, (1964).

11. L. HORMANDER, Linear Partial Differential Operators, Springer, (1963).

12. F. JOHN, Partial Differential Equations, Springer, New York, (1982).

13. J. LERAY, Hyperbolic Equations, Lectures at the Institute for Advanced Studies, Princenton, (1956).

14. S. G. MIKHLIN, Mathematical Physics, An Advanced Course, North-Holland, (1970).

15. I. G. PETROVSKY, On Some Problems of Partial Differential Equations, Usp. Mat. Nauk. 1, 3-4 (1314), 44-70, (1946), Amer. Math. Translations, 12.

16. I. G. PETROVSKY, Lectures on Partial Differential Equations, Interscience Publisher, New York, (1954).

17. W. A. STRAUSS, Particl Differential Equation, An Introduction, John Wiley \& Sons, Inc., (1992). 